\title{
Professionalisierung \\ für inklusiven Unterricht im Rahmen \\ interdisziplinärer und videogestützter Lehrveranstaltungen
}

\author{
Ann-Christin Faix, Elke Wild, Birgit Lütje-Klose \& Annette Textor \\ Journal für Psychologie, 27(2), 71-94 \\ https://doi.org/10.30820/0942-2285-2019-2-71 \\ www.journal-fuer-psychologie.de
}

\section{Zusammenfassung}

Der Beitrag stellt ein videobasiertes Lehrkonzept vor, das in interdisziplinärer Kooperation entwickelt und durchgeführt worden ist. Mit der Lehrveranstaltung wird das Ziel verfolgt, die auf inklusiven Unterricht bezogene professionelle Wahrnehmung von Studierenden zu fördern. Um dies zu erreichen, arbeiten die Studierenden zunächst ihre Subjektiven Theorien über guten inklusiven Unterricht heraus, die potenziell im Widerspruch zu bildungswissenschaftlichem Wissen stehen. Da es nicht nur eine mögliche Perspektive auf guten inklusiven Unterricht gibt, werden im Seminar die Perspektiven der Pädagogischen Psychologie, der Schulpädagogik und der Sonderpädagogik integrativ vermittelt. Die Teilnehmer*innen operationalisieren die Befunde und überführen sie in ein Raster zur Beobachtung von inklusivem Unterricht. Das Raster kommt schließlich bei Videoanalysen und Unterrichtshospitationen zum Einsatz. Das Seminar wurde hinsichtlich verschiedener Variablen wie inklusionsbezogenen Einstellungen und bildungswissenschaftlichem Wissen positiv evaluiert.

Schlüsselwörter: Inklusiver Unterricht, professionelle Unterrichtswahrnehmung, inklusionsbezogene Einstellungen, bildungswissenschaftliches Wissen, Unterrichtsvideos, Interdisziplinarität

\section{Summary}

Professionalization for teaching in inclusive classrooms within interdisciplinary video-based seminars

This article presents the conceptualization of an interdisciplinary video-based seminar implemented in teacher education at university. The seminar aims at promoting students' professional vision of teaching in inclusive classrooms. As a first step towards this goal, students map out and reflect on their subjective theories about what they consider high quality 
teaching in inclusive classrooms, which may contradict findings from educational and psychological research. To highlight different disciplinary perspective on high quality teaching in inclusive classrooms, the seminar explicitly integrates perspectives from school pedagogy, special education, and psychology. Next, these perspectives are used to develop a grid helping students to systematically observe teaching in inclusive classrooms. Finally, the grid is used in video analyses and real-life observations of inclusive classrooms. The evaluation of the seminar showed positive changes with regard to several variables such as attitudes towards inclusive education and educational knowledge.

Key words: teaching in inclusive classrooms, professional vision, attitudes towards inclusive education, educational knowledge, video, multidisciplinarity

\section{$1 \quad$ Einleitung}

Im vorliegenden Beitrag werden ein interdisziplinäres und videobasiertes Lehrkonzept zur Förderung inklusionsbezogener Kompetenzen von Lehramtsstudierenden sowie ausgewählte Evaluationsergebnisse vorgestellt. Das Seminar wurde in interdisziplinärer Kooperation von Vertreterinnen der Pädagogischen Psychologie, der Schulpädagogik und der Sonderpädagogik an der Universität Bielefeld erarbeitet und durchgeführt. Es richtet sich an Studierende der Lehrämter für Gymnasien und Gesamtschulen sowie Haupt-, Real-, Sekundar- und Gesamtschulen, um inklusionssensible Inhalte dem Beschluss der Kultusminister- und Hochschulrektorenkonferenz gemäß in allen Lehramtsstudiengängen curricular zu verankern (KMK und HRK 2015). Ziel der Intervention ist die Förderung einer inklusionsbezogenen professionellen Wahrnehmung der Studierenden (Barth 2016; Seidel, Blomberg und Stürmer 2010; Seidel und Stürmer 2014; Sherin 2007; Sherin und van Es 2009). Die professionelle Wahrnehmung baut auf dem bildungswissenschaftlichen Wissen der Studierenden auf und trägt im Sinne handlungsleitender Schemata dazu bei, relevante Unterrichtssituationen wahrzunehmen, diese wissensbasiert zu reflektieren und zu bewerten (ebd.). Das Seminar knüpft an die Subjektiven Theorien der Teilnehmer*innen an, um diese an bildungswissenschaftliches Wissen anzunähern (Wahl 2013). Auf diese Weise soll trägem Wissen vorgebeugt und professionelles (evidenzbasiertes) Handeln und Urteilen angebahnt werden (ebd.). Die Lehrveranstaltung wurde quasi-experimentell (Prä-Post-Follow-upDesign mit Kontrollgruppe) positiv evaluiert. Im Fokus dieses Beitrags stehen Analysen zu der Frage, ob und inwieweit das Seminar zur Förderung der inklusionsbezogenen Einstellungen und des bildungswissenschaftlichen Wissens der Teilnehmenden beiträgt. Die Befunde werden abschließend kritisch und mit Blick auf Konsequenzen für die Weiterentwicklung des Lehrkonzepts diskutiert. 


\section{Theoretischer Hintergrund und Forschungsstand}

\subsection{Inklusionsbegriff}

Im Zuge der Ratifizierung der UN-Behindertenrechtskonvention hat sich die Bundesrepublik Deutschland verpflichtet, ein inklusives Bildungssystem auf allen Ebenen zu etablieren, um allen Menschen einschließlich denjenigen mit Behinderungen Partizipation zu ermöglichen (UN-BRK 2008). In Artikel 24 der UN-Konvention werden die Rechte von Schüler*innen mit » special educational needs «, also besonderen pädagogischen Unterstützungsbedarfen aufgrund von Behinderungen und Benachteiligungen, auf eine inklusive Erziehung und Bildung sowie die Schaffung » angemessener Vorkehrungen « zur Berücksichtigung entsprechender Bedarfe in allen Bildungsinstitutionen besonders hervorgehoben. Allerdings ist der Anspruch auf eine inklusive Beschulung im Sinne der UN-BRK nicht auf diese Gruppe beschränkt, sondern im Sinne eines weiten Inklusionsverständnisses und einer »education for all $\ll \mathrm{zu}$ verstehen, wie in den Zusatzprotokollen betont wird: »Alle Menschen haben ein Recht auf inklusive Bildung - nicht nur Menschen mit Behinderungen « (Deutsches Institut für Menschenrechte 2017, 1). Die Empfehlungen der deutschen Kultusministerkonferenz und die Schulgesetze der Länder sind dagegen im Sinne eines engen, auf die Differenzkategorie sonderpädagogischer Förderbedarf bezogenen Inklusionsverständnisses formuliert (KMK und HRK 2015; SchulG NRW).

Die Frage nach dem zugrunde gelegten Inklusionsverständnis (zsf. Lindmeier und Lütje-Klose 2018; Piezunka, Gresch und Wrase 2018) wird im Seminar explizit aufgegriffen, da es für die Entwicklung inklusionsbezogener Einstellungen, Zuständigkeitswahrnehmungen und Handlungsorientierungen von (angehenden) Lehrkräften als hoch bedeutsam anzusehen ist (Kullmann, Lütje-Klose, Textor, Berard und Schitow 2015; Moser, Kuhl, Redlich und Schäfer 2014). Dabei ist die Differenzlinie Behinderung insofern besonders relevant, als sich damit spezifische, den Studierenden der allgemeinen Lehrämter vielfach nicht geläufige Unterstützungsbedarfe verbinden können, die ggf. Abwehr gegenüber den als fremd und anders empfundenen Schüler*innen, Überforderungsängste und eingeschränkte Selbstwirksamkeitsüberzeugungen nach sich ziehen können (ebd.; Loreman, Earle, Sharma und Forlin, 2007; Urton, Wilbert und Hennemann, 2015). Im Seminar wird daher zunächst ein enges, auf die inklusive Beschulung von Schüler*innen mit ausgewiesenem sonderpädagogischem Förderbedarf bezogenes Inklusionsverständnis eingeführt. Dieses enge Inklusionsverständnis wird jedoch fortlaufend immer wieder problematisiert und mit Blick auf weitere benachteiligende Faktoren dekonstruiert, sodass auf ein weites, auf weitere Heterogenitätsdimensionen bezogenes Inklusionsverständnis hingearbeitet wird (Textor, Kullmann und Lütje-Klose 2014). 


\subsection{Bildungswissenschaftliches Wissen als Basis professioneller Unterrichtswahrnehmung}

Um inklusive Lerngruppen erfolgreich unterrichten zu können, müssen (angehende) Lehrkräfte Kompetenzen für den Umgang mit inklusiven Lerngruppen erwerben (Lütje-Klose, Miller und Ziegler 2014; Moser 2018). Im beschriebenen Seminar sollen Kompetenzen der Studierenden in Form der professionellen Unterrichtswahrnehmung, die professionelles Wissen und Handeln systematisch miteinander verknüpft, gefördert werden (Barth 2016; Seidel et al. 2010; Seidel und Stürmer 2014; Sherin 2007; Sherin und van Es 2009). In den häufig rezipierten Kompetenzmodellen wird neben dem Fachwissen und dem fachdidaktischen Wissen das pädagogisch-psychologische Wissen als Kern professioneller Kompetenz ausgewiesen (z. B. COACTIV, Kunter et al. 2011). Diese Konstruktion ist jedoch verkürzt, stellt die pädagogisch-psychologische Sicht - gerade wenn es um inklusiven Unterricht geht - nur eine unter weiteren möglichen Perspektiven dar. Aus diesem Grund erscheint es adäquat, statt von pädagogischpsychologischem Wissen von bildungswissenschaftlichem Wissen (z. B. BilWiss, KuninaHabenicht et al. 2012; Terhart et al. 2012) zu sprechen. An diese Überlegungen anknüpfend ist das hier beschriebene Seminar interdisziplinär angelegt und bezieht zur Förderung der professionellen Unterrichtswahrnehmung pädagogisch-psychologische, schulpädagogische und sonderpädagogische Perspektiven auf guten inklusiven Unterricht gleichermaßen ein (vgl. Abschnitt 3.1). Professionelles (bildungswissenschaftliches) Wissen stellt dabei die Voraussetzung für die Wahrnehmung und wissensgeleitete Interpretation relevanter Unterrichtssituationen dar. Die Wahrnehmung relevanter Unterrichtssituationen umfasst die sensorische Erfassung der Umwelt sowie einen Abgleich mit (bildungswissenschaftlichem) Wissen, um Tiefen- und Oberflächenstrukturen von Unterricht identifizieren zu können. Im Rahmen der wissensgeleiteten Interpretation und Beurteilung werden die wahrgenommenen Unterrichtsmerkmale vor dem Hintergrund des (bildungswissenschaftlichen) Wissens bewertet und Schlussfolgerungen für die Qualität des beobachteten Lehrer*innenhandelns gezogen (Barth 2016). Darauf basierend werden Handlungsalternativen abgeleitet, aus denen schließlich eine ausgewählt und im Unterricht handelnd umgesetzt wird (ebd.).

\subsection{Subjektive Theorien und inklusionsbezogene Einstellungen}

Um angehende Lehrkräfte für inklusiven Unterricht zu professionalisieren, sind neben dem bildungswissenschaftlichen Wissen auch Subjektive Theorien und inklusionsbezogene Einstellungen $\mathrm{zu}$ adressieren. In der bildungswissenschaftlichen Literatur werden in Bezug auf verwandte Konstrukte oftmals die Begriffe Beliefs und Überzeugungen ver- 
wendet (Reusser und Pauli 2014). Subjektive Theorien sind wie Einstellungen unter bestimmten Bedingungen handlungsleitend und werden in der Forschung als mental repräsentierte semantische Netze beschrieben (Groeben, Wahl, Schlee und Scheele 1988; Wahl 2013). Im Gegensatz zu Einstellungen werden Subjektive Theorien als kognitive Strukturen der Selbst- und Weltsicht mit zumindest impliziter Argumentationsstruktur konzipiert (vgl. Groeben et al. 1988, 19). Insofern beeinflussen sie, wie Lehrkräfte Unterricht wahrnehmen, planen und durchführen. Um potenzielle Widersprüche zwischen den Subjektiven Theorien und bildungswissenschaftlichem Wissen möglichst zu minimieren, müssen Subjektive Theorien reflektiert und mit wissenschaftlichen Theorien und Forschungsergebnissen abgeglichen werden (Wahl 2013; Wilde und Kunter 2016).

Subjektive Theorien beinhalten in der Regel auch wertbehaftete Einstellungen, welche die Wahrnehmung und das Handeln von Lehrkräften unter bestimmten Bedingungen leiten. In der Sozialpsychologie werden Einstellungen (attitudes) als psychologische Tendenz, eine Entität mit einem bestimmten Maß an Zustimmung oder Ablehnung zu bewerten, verstanden. Die Bewertung kann sowohl eine kognitive, affektive als auch behaviorale Komponente aufweisen (Eagly und Chaiken 1993; Ruberg und Porsch 2017). In einer Übersicht über quantitative Studien zu inklusionsbezogenen Einstellungen von (angehenden) Lehrkräften identifizieren Ruberg und Porsch (2017) Erfahrungen, die (angestrebte) Schulform, die Art der Behinderung bzw. das Heterogenitätsmerkmal, die Selbstwirksamkeit sowie den jeweiligen Erhebungsort als wesentliche Einflussfaktoren auf die inklusionsbezogenen Einstellungen. Die Forschungslage zum Zusammenhang von Überzeugungen bzw. Einstellungen und Handeln wiederum ist weitgehend uneinheitlich. In vielen Studien konnte bereits ein Einfluss von Überzeugungen auf das Handeln von Lehrkräften empirisch nachgewiesen werden, in anderen Fällen gelingt ein solcher Nachweis nicht (Fives und Buehl 2012; Wilde und Kunter 2016). Dies gilt ähnlich in Bezug auf inklusiven Unterricht: Inklusionsbezogene Einstellungen gelten als Basis für die erfolgreiche Implementation inklusiver Unterrichtspraktiken, wobei der Einfluss von Einstellungen auf das Handeln von Lehrkräften noch empirisch nachzuweisen ist (Ruberg und Porsch 2017; Moser 2018). Als Erklärung für nicht nachgewiesene Zusammenhänge ziehen Wilde und Kunter (2016) neben methodischen Herausforderungen die Theorie geplanten Verhaltens (Ajzen 2012) heran: Handeln ist abhängig von Überzeugungen und Einstellungen, wird jedoch gleichsam von weiteren Faktoren, wie wahrgenommener Verhaltenskontrolle, beeinflusst.

Das in diesem Beitrag beschriebene Lehrkonzept hat zum Ziel, Studierende durch die Förderung von Kompetenzen im Bereich der professionellen Unterrichtswahrnehmung für inklusiven Unterricht zu professionalisieren. Ein multiperspektivisches bildungswissenschaftliches Wissen bildet die Basis professioneller Unterrichtswahrnehmung, um relevante Unterrichtssituationen wahrnehmen und wissensbasiert interpretieren zu können. 


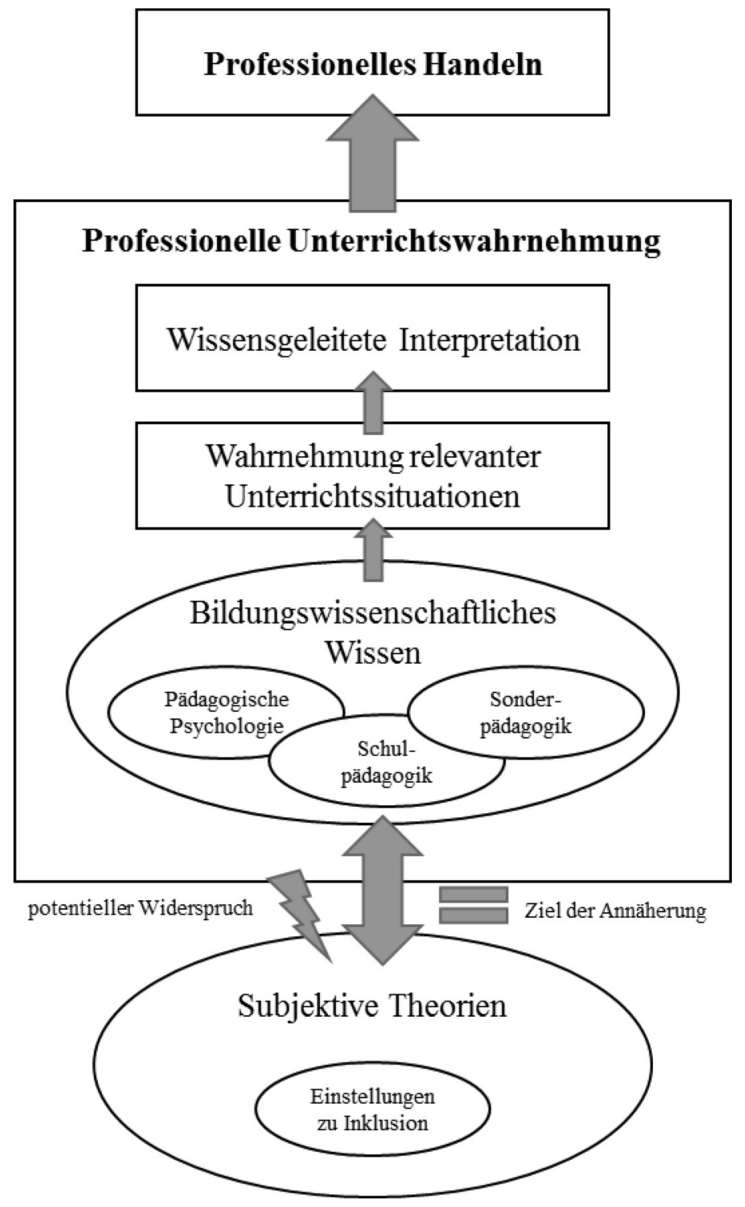

Abbildung 1:Zusammenhang von Subjektiven Theorien, Einstellungen, bildungswissenschaftlichem Wissen und professioneller Unterrichtswahrnehmung (eigene Darstellung)

Handlungsleitende Subjektive Theorien und Einstellungen der Studierenden können möglicherweise stark von bildungswissenschaftlichem Wissen abweichen. Aus diesem Grund bezieht das Seminar die Subjektiven Theorien und Einstellungen mit ein, um eine Annäherung an bildungswissenschaftliches Wissen zu unterstützen. Dabei soll in Anlehnung an die UN-BRK ein weites Inklusionsverständnis, das neben sonderpädagogischem Förderbedarf auch weitere im schulischen Kontext relevante Differenzlinien einbezieht, angebahnt werden. 


\section{Hochschuldidaktische Maßnahmen}

Vor dem Hintergrund der vorangegangen Ausführungen wird im Folgenden erläutert, mit welchen hochschuldidaktischen Maßnahmen die beschriebenen Ziele einzulösen versucht werden.

\subsection{Interdisziplinäres Teamteaching zur Überwindung trägen Wissens}

Durch die Aufteilung der Lehrer*innenbildung entlang verschiedener Fächer und Phasen wird professionsrelevantes Wissen weitgehend isoliert vermittelt, sodass bei unzureichender Integration die Gefahr trägen Wissens droht (Hellmann 2019). Dieser Praxis gegenüber beschreibt der Ansatz der Kobärenz die systematische Verknüpfung von Inhalten, Strukturen und Phasen in der Lehrer*innenbildung (ebd.). Im Freiburger Säulen-Phasen-Modell der Kohärenz (ebd.) werden verschiedene Wissensdomänen (Fachwissen, fachdidaktisches Wissen, bildungswissenschaftliches Wissen) und Praxiserfahrungen als Säulen dargestellt und verschiedene Phasen des Professionalisierungsprozesses (Bachelor- und Masterstudium, Referendariat, Beruf) abgebildet. Es wird davon ausgegangen, dass Verknüpfungen sowohl horizontal zwischen den Domänen als auch vertikal - phasenübergreifend - innerhalb der Domänen möglich sind (ebd.). Das in diesem Beitrag beschriebene Seminar stellt durch interdisziplinäres Teamteaching horizontale Kohärenz innerhalb der Bildungswissenschaften her, indem die Perspektiven der Pädagogischen Psychologie, der Schulpädagogik und der Sonderpädagogik miteinander verknüpft werden. Interdisziplinäre Lehrveranstaltungen können dazu beitragen,

$>$ die Isolation zwischen den Disziplinen zu reduzieren (Smith Ducoffe, Tromley und Tucker 2006).

$>$ Wissen problembezogen zu integrieren, anstatt Informationen lediglich getrennt zu verarbeiten (Daum und Schneider 2006).

$>$ einen kritischen und multiperspektivischen Blick auf den jeweiligen Lerngegenstand zu entwickeln (Lattuca, Voight und Fath 2004; Smith Ducoffe et al. 2006).

$>$ zu motivieren und Sinn zu stiften, indem bedeutsame Aspekte in den Fokus gerückt werden (ebd.).

$>\quad$ das Reflexionsvermögen zu stärken (Smith Ducoffe et al. 2006).

Der Beitrag der beteiligten Disziplinen kann - stark verkürzt - folgendermaßen zusammengefasst werden: Die Pädagogische Psychologie richtet ihren Fokus vor allem auf die Lernwirksamkeit von Unterricht und Unterrichtsmethoden. Diese Fragen werden von der Schulpädagogik zwar ebenfalls aufgegriffen, aber um Fragen der Didaktik, das 
heißt der Auswahl und Vermittlung von Unterrichts- und Bildungsinhalten, erweitert. In den Perspektiven der Sonderpädagogik und der inklusiven Pädagogik rückt schließlich die Frage in den Mittelpunkt, wie die Unterrichts- und Bildungsinhalte so ausgewählt, aufbereitet und vermittelt werden können, dass alle Schüler*innen, auch solche mit besonderen individuellen Lernausgangslagen, bestmöglich individuell gefördert werden und sowohl inhaltlich als auch sozial partizipieren können. Inhaltlich gibt es eine Vielzahl von Überschneidungen zwischen den disziplinären Perspektiven, sodass sie nicht trennscharf gegeneinander abgegrenzt werden können. Die Integration wurde im Seminar im interdisziplinären Teamteaching realisiert und soll im Folgenden anhand zweier Beispiele konkret veranschaulicht werden:

Die Bedeutung des Konstruktes Klassenführung wird in jeder der drei Perspektiven betont. Bei Kindern, die als hyperaktiv wahrgenommen werden, erweist sich der in der Praxis häufig vorzufindende Einsatz von Token-Programmen (Perspektive der Pädagogischen Psychologie) für diese Gruppe oftmals als unzureichend. Hier sind weiterführende Maßnahmen mit Blick auf die Organisation der Lernumgebung und die pädagogische Beziehungsgestaltung zu ergreifen, die durch die Perspektive der Sonderpädagogik und der Schulpädagogik in jeweils spezifischer Form eingebracht werden.

Auch die individuelle Förderung von Kindern spielt in jeder der Disziplinen eine Rolle. Aus der Perspektive der Sonderpädagogik und der inklusiven Pädagogik wird dabei zudem die Bedeutung eines gemeinsamen Unterrichtsgegenstandes betont und gefordert, dass Individualisierung in Gemeinsamkeit stattfinden solle (Werning und Lütje-Klose 2016).

\subsection{Verknüpfung von Wissen und Wahrnehmung durch Unterrichtsvideos}

Zur Förderung der professionellen Unterrichtswahrnehmung erweisen sich Unterrichtsvideos als vorteilhaft und zielführend, da inklusive Unterrichtspraxis ohne Handlungsdruck erfahrbar wird (Goeze 2010; Gold, Förster und Holodynski 2013). Positive Effekte von videobasierten Lernsettings auf die professionelle Wahrnehmung konnten bereits empirisch belegt werden (z.B. Gold et al. 2013; Hellermann, Gold und Holodynski 2015). Durch die Analyse > modellhafter< Unterrichtssituationen können neue Unterrichtsideen entwickelt und Einstellungen bearbeitet werden, während am Beispiel > kritischer $<$ Situationen alternative Handlungsoptionen entwickelt und Diagnose- und Urteilskompetenzen gefördert werden können (Petko, Prasse und Reusser 2014). Durch die Kontrastierung > modellhafter $<$ und > kritischer $<$ Unterrichtspraxis sollen somit zielführende, aber auch ineffiziente Unterrichtspraktiken aufgezeigt und so die Praxisrelevanz der vermittelten Konzepte und Theorien unmittelbar erfahrbar gemacht werden. Mit der Auswahl der Videos sind, gerade wenn es um die Wahrnehmung 
und Beurteilung inklusiven Unterrichts geht, vier grundlegende Herausforderungen verbunden. Die Praxiseinsichten sollten so angelegt sein, dass sie

$>$ den Studierenden einen realistischen Eindruck von der Komplexität pädagogischen Handelns vermitteln, ohne die Studierenden zu überfordern und inklusionsskeptische Einstellungen damit zu verstärken (Goeze 2010).

$>\quad$ >good practice «-Beispiele aufzuzeigen, ohne diese als universale Handlungsmuster für beliebige Situationen zu präsentieren.

$>$ den Nutzen einer interdisziplinären Betrachtung aufzeigen, ohne die zwischen den wissenschaftlichen Perspektiven angelegten Spannungsfelder zu negieren (vgl. Abschnitt 3.1).

$>$ die Bedeutung allgemeindidaktischer Analysen verdeutlichen, ohne den Stellenwert fachdidaktischer Analysen zu vernachlässigen (vgl. Abschnitt 8).

\section{3 Überführen theoretischer Inputs in ein Urteilsraster}

Im Anschluss an jeden der theoretischen Inputs sind die Teilnehmer*innen aufgefordert, relevante Qualitätsmerkmale zu operationalisieren und in ein sukzessiv zu vervollständigendes Raster für die Beurteilung inklusiven Unterrichts zu überführen. Hier wird ein Anreiz für die vertiefende Auseinandersetzung mit den vermittelten Inhalten gesetzt, die an dem studentischen Interesse nach praxisnaher Ausbildung ansetzt. Das Raster wird im weiteren Verlauf des Seminars stetig überarbeitet und weiterentwickelt, sodass es die einzelnen Phasen des Seminars wie ein roter Faden miteinander verbindet. Während der Videoanalysen werden die einzelnen Items erprobt, ausgeschärft oder teilweise auch wieder verworfen. ${ }^{1}$ Professionelles Wissen wird situiert und praxisorientiert erworben und soll durch den Einsatz des Rasters bei Videoanalysen gefördert werden, indem Begriffe und Konzepte mit unterrichtspraktischen Beispielen verknüpft werden (Seidel und Thiel 2017).

\subsection{Hospitationen zur selbstkritischen Prüfung Subjektiver Theorien und inklusionsbezogener Einstellungen}

Das finalisierte Urteilsraster kommt bei Hospitationen in ortsansässigen »good practice «-Schulen zu Einsatz. Bei der Beobachtung eines Unterrichts, der in hohem Maße von den im Seminar erarbeiteten Qualitätsmerkmalen abweicht, droht die Gefahr einer Verstärkung inklusionsskeptischer Einstellungen. Um dem vorzubeugen, werden Schulen ausgewählt, die über langjährige Erfahrungen mit inklusivem Unterricht verfügen und mit denen bereits zuvor Kooperationen bestanden. Auf diese Weise wird versucht sicherzustellen, dass die Studierenden während ihrer Hospitationen guten inklusiven 
Unterricht in dem im Seminar vertretenen Sinne beobachten können. Die Hospitationen dienen der Verständniskontrolle der in das Raster überführten Qualitätsmerkmale. Zudem sollen durch die Beobachtung gelingender inklusiver Unterrichtspraxis positive stellvertretende Unterrichtserfahrungen gesammelt werden, über welche ein positiver Einfluss auf die inklusionsbezogenen Einstellungen der Studierenden und eine selbstkritische Überprüfung derselben angestrebt werden (z. B. Avramidis und Kalyva 2007; Bosse und Spörer 2014; Hellmich und Görel 2014). Durch die Hospitationen wird das erworbene bildungswissenschaftliche Wissen zusätzlich mit schulpraktischen Erfahrungen verknüpft, sodass die horizontale Kohärenz weiter ausgebaut wird (Hellmann 2019).

\subsection{Strukturlegetechnik als Instrument zur eigenverantwortlichen Einschätzung des erzielten Lernfortschritts}

Die Studierenden reflektieren ihre Subjektiven Theorien über guten inklusiven Unterricht in der ersten und letzten Seminarsitzung mithilfe einer Strukturlegetechnik (Faix, Lütje-Klose, Textor und Wild 2019a; Scheele, Groeben und Christmann 1992). Die Inhalte der Subjektiven Theorie werden durch frei wählbare Begriffe auf Klebezetteln repräsentiert und die Beziehungen zwischen den Inhalten werden durch Pfeile zwischen den Begriffen visualisiert. Durch die Strukturlegepläne legen die Studierenden ihre Subjektiven Theorien gewissermaßen sichtbar vor sich auf dem Tisch aus (Wahl 2001). Auf diese Weise können sie Argumentationslücken und Widersprüche in ihren Subjektiven Theorien identifizieren, bearbeiten und reflektieren. Am Ende des Seminars haben sie die Möglichkeit, einen neuen Strukturlegeplan zu erstellen oder ihren alten Strukturlegeplan zu überarbeiten. Auf diese Weise gleichen sie ihre Subjektiven Theorien zu Beginn und am Ende des Seminars miteinander ab und gelangen so zu einer eigenverantwortlichen Einschätzung ihres erzielten Lernfortschritts (Faix et al. 2019a).

\section{$4 \quad$ Fragestellung}

An der Universität Bielefeld wurde im Rahmen der wissenschaftlichen Maßnahme »Professionalisierung durch die Konfrontation Subjektiver und wissenschaftlicher Theorien über guten inklusiven Unterricht im Rahmen videogestützter Lehrveranstaltungen « ein Seminar entwickelt, erprobt und evaluiert, in welchem die zuvor beschriebenen Maßnahmen realisiert wurden. Die Maßnahme ist Teil des Bielefelder Projektes Biprofessional, das im Rahmen der Qualitätsoffensive Lehrerbildung gefördert wird. ${ }^{2}$ Es wird untersucht, ob durch das Seminar die angestrebten Veränderungen des bildungswissenschaftlichen Wissens und inklusionsbezogener Einstellungen erzielt werden können. 


\section{Methodik}

\subsection{Konzeption und Verortung des interdisziplinären Seminars}

Das Seminar wurde von Vertreterinnen der Pädagogischen Psychologie, der Schulpädagogik und der Sonderpädagogik konzipiert, in einem ersten Durchlauf im Wintersemester 2016/17 pilotiert und auf Grundlage der Evaluationsergebnisse weiterentwickelt und im Wintersemester 2017/18 summativ evaluiert. Die Lehrveranstaltung umfasst vier Semesterwochenstunden und ist in den bildungswissenschaftlichen Anteilen der Lehramtsstudiengänge Gymnasium und Gesamtschule sowie Haupt-, Real-, Sekundarund Gesamtschule im Modul »Erziehen und Unterrichten « verortet. Ziel des Moduls ist es, angehende Lehrkräfte für die Komplexität des pädagogischen Handlungsfeldes Schule zu sensibilisieren. Um den Veränderungen einer sich inklusiv entwickelnden Schullandschaft Rechnung zu tragen, sollen in diesem Modul inklusionspädagogische Basiskompetenzen vermittelt werden (Lütje-Klose, Neumann und Streese 2017). Den Studierenden wird empfohlen, das Seminar im vierten oder fünften Semester des Bachelorstudiums zu besuchen. Teil des Moduls ist auch eine Praxisstudie, welche die Studierenden möglichst im Anschluss an das hier beschriebene Seminar absolvieren (https://ekvv.uni-bielefeld.de/sinfo/publ/modul/26786937). Die Veranstaltung wurde von zwei Hochschullehrerinnen aus der Pädagogischen Psychologie und der Sonderpädagogik, die über vergleichbar langjährige Erfahrungen in der universitären Lehre verfügen, im Teamteaching geleitet. Das Seminar umfasst vier Phasen:

1) Interdisziplinäre Inputs und Arbeit am Urteilsraster im Wechsel

2) Erprobung und Weiterentwicklung des Rasters anhand von Videoanalysen

3) Anwendung des Rasters in Hospitationen

4) Nachträgliche Reflexion des Instruments, seiner Kategorien und der Hospitations- und Praktikumserfahrungen in einer Reflexionssitzung zwei Monate nach dem Ende des Seminars.

\subsection{Forschungsdesign}

Das Lehrkonzept wurde auf der Grundlage eines quasi-experimentellen Prä-Post-Followup-Designs mit einer Kontrollgruppe (KG) evaluiert. Die Messungen erfolgten in der ersten $\left(t_{1}\right)$ und letzten Seminarsitzung $\left(t_{2}\right)$. Die Reflexionssitzung zwei Monate nach dem Ende des Seminars wurde zur Erhebung der Follow-up-Daten $\left(t_{3}\right)$ genutzt. Die Teilnehmer*innen der KG konnten für diesen Termin nicht gewonnen werden, sodass die Followup-Daten nur für die Experimentalgruppe (EG) vorliegen. Als KG diente eine Lehrveranstaltung im gleichen Modul. In der KG kamen ebenfalls Videoanalysen und Hospitationen 
zum Einsatz, allerdings wurde auf die Durchführung im interdisziplinären Teamteaching und auf die Erstellung eines eigenen Urteilsrasters verzichtet. Die Zuweisung zu den Seminaren erfolgte zentral über das elektronische Teilnehmermanagement der Universität.

\subsection{Stichprobe}

Es liegen die Daten von 14 Teilnehmer*innen der EG für drei Messzeitpunkte vor. Damit ist die EG deutlich kleiner als die 44 Teilnehmer*innen umfassende KG. Möglicherweise versuchen die Studierenden aufgrund des hohen Arbeitsaufwands (Erstellung des Urteilsrasters, Hospitationen, Reflexionssitzung) bevorzugt einen Platz in anderen Lehrveranstaltungen zu erhalten, sodass die EG im Vergleich zu anderen Seminaren weniger Teilnehmer*innen umfasst. Hinsichtlich der studierten Fachsemester sind beide Gruppen miteinander vergleichbar. Auch sind in beiden Gruppen der Anteil der weiblichen Teilnehmerinnen sowie der Anteil der Teilnehmenden des Lehramtes für Gymnasien und Gesamtschulen höher. Die Studiengänge unterscheiden sich dem Lehrerausbildungsgesetz des Landes Nordrhein-Westfalen gemäß vor allem darin, dass für das Lehramt an Haupt-, Real-, Sekundar- und Gesamtschulen mehr bildungswissenschaftliche Module absolviert werden müssen. So umfasst das Studium der Bildungswissenschaften im Bachelorstudiengang für das Lehramt an Haupt-, Real-, Sekundarund Gesamtschulen 60 Leistungspunkte, im Studiengang für das Lehramt an Gymnasien und Gesamtschulen 30 Leistungspunkte.

\begin{tabular}{|c|c|c|c|}
\hline & $\begin{array}{l}\text { Experimental- } \\
\text { gruppe (EG) }\end{array}$ & $\begin{array}{l}\text { Kontroll- } \\
\text { gruppe (KG) }\end{array}$ & Gesamt \\
\hline Teilnehmer*innen $(n)$ & 14 & 44 & 58 \\
\hline Fachsemester (M, (SD)) & $4.9(2.1)$ & $4.8(1.3)$ & $4.8(1.5)$ \\
\hline \multicolumn{4}{|l|}{ Geschlecht } \\
\hline Weiblich (n) & 12 & 27 & 39 \\
\hline Männlich (n) & 2 & 17 & 19 \\
\hline \multicolumn{4}{|l|}{ Lehramt } \\
\hline Gymnasium und Gesamtschule ( $n$ ) & 8 & 27 & 35 \\
\hline Haupt-, Real-, Gesamt- und Sekundarschule ( $n$ ) & 3 & 16 & 19 \\
\hline $\begin{array}{l}\text { Haupt-, Real-, Gesamt- und Sekundarschule } \\
\text { mit Integrierter Sonderpädagogik ( } n \text { ) }\end{array}$ & 2 & 1 & 3 \\
\hline Andere $(n)$ & 1 & 0 & 1 \\
\hline
\end{tabular}

Tabelle 1: Stichprobenbeschreibung 


\subsection{Instrumente}

Um das bildungswissenschaftliche Wissen standardisiert zu erfassen, wurden Teile des PUW eingesetzt (Pädagogisches Unterrichtswissen, König und Blömeke 2009). Pädagogisches Wissen wird im PUW nicht als homogenes, sondern als mehrdimensionales Konstrukt modelliert, das sich aus den Dimensionen Strukturierung von Unterricht, Motivierung, Umgang mit Heterogenität, Klassenführung und Leistungsbeurteilung zusammensetzt (ebd.). Diese Dimensionen wurden aus der Wissenstopologie von Shulman (1986), den KMK-Standards (2004) sowie Standardwerken der Allgemeinen Didaktik und Unterrichtsforschung abgeleitet und sollen damit den berufsbezogenen Anforderungen von Lehrkräften entsprechen (König und Blömeke 2009). Die Autor*innen betonen, dass das Instrument einen Bezug zu zentralen Lehrschwerpunkten der am Lehramtsstudium beteiligten Disziplinen Erziehungswissenschaft, Psychologie und Soziologie herstellt, indem es Fragen der Unterrichtsplanung, der Lernzielkontrolle und den Umgang mit sozialer Ungleichheit berücksichtigt (ebd.). ${ }^{3} \mathrm{Da}$ das beschriebene Seminar auf die Vermittlung einer multiperspektivischen Wissensbasis abzielt, erscheint das PUW geeignet, das im Seminar vermittelte Wissen weitgehend, aber nicht vollständig, abzubilden (vgl. Abschnitt 3.1). ${ }^{4}$

Das Instrument umfasst offene und geschlossene Antwortformate, um sowohl deklaratives als auch prozedurales Wissen erfassen zu können. Zur Auswertung liegt ein Manual vor (König und Blömeke 2010). Beim Einsatz des vollständigen PUW wären maximal 58 Punkte erreichbar gewesen, aus zeitökonomischen Gründen konnten bei der Testung jedoch nur Aufgaben im Umfang von 40 Punkten berücksichtigt werden. Davon entfielen vier Punkte auf die Dimension Strukturierung von Unterricht, 14 Punkte auf Umgang mit Heterogenität, fünf Punkte auf Klassenführung, fünf Punkte auf Leistungsbeurteilung sowie zwölf Punkte auf den Bereich Motivierung. Ausgespart wurden solche Aufgaben, die inhaltlich deutlich über den üblichen curricularen Anspruch eines Bachelorseminars hinausgehen (z. B. die Gliederung eines schriftlichen Unterrichtsentwurfs). Die offenen Aufgabenformate wurden von zwei unterwiesenen Rater*innen mithilfe des Kodiermanuals unabhängig ausgewertet. Als Maß zur Bestimmung der Interraterreliabilität wurde das gewichtete Cohens Kappa herangezogen. Der Wert $\kappa=0,79$ zeigt eine starke Übereinstimmung der Rater*innen (Wirtz und Caspar 2002).

Des Weiteren wurden zur Messung der inklusionsbezogenen Einstellungen die Subskalen Attitudes (positive Haltungen) und Concerns (Bedenken) des SACIE-R (Sentiments, Attitudes and Concerns about Inclusive Education Revised) in ihrer deutschen Übersetzung verwendet (Feyerer et al. 2013). Die Subskala Sentiments wurde bei der Untersuchung nicht eingesetzt, da diese keinen direkten Bezug zu inklusivem Unterricht aufweist, sondern stattdessen grundsätzliche Haltungen gegenüber Beein- 
trächtigungen und Behinderungen misst. Innerhalb der Subskala Concerns wurden die Begriffe »Beeinträchtigungen « und »Behinderungen « jeweils durch den Begriff » sonderpädagogischer Förderbedarf « ersetzt, da in der Lehrveranstaltung vorwiegend mit dieser Kategorie operiert wurde und durch die Klarheit der Begrifflichkeiten die Genauigkeit der Messung erhöht werden sollte. Beide Subskalen bestehen aus jeweils fünf Items, die auf einer vierstufigen Likertskala entlang der Antwortformate stimme nicht zu, stimme eher nicht $z u$, stimme eher $z u$ sowie stimme $z u$ eingeschätzt wurden. Die Items der Subskala Concerns weisen gegenüber der Subskala Attitudes eine negative Polung auf. Höhere Skalenwerte der Subskala Concerns entsprechen somit höheren inklusionsbezogenen Bedenken, während hohe Ausprägungen der Subskala Attitudes einer aufgeschlossenen inklusionsbezogenen Einstellung entsprechen. Zur Berechnung der Reliabilität beider Subskalen wurde das Maß Cronbachs Alpha verwendet, wobei der Wert für die Subskala Attitudes im guten Bereich $(\alpha=0,81)$ und für die Subskala Concerns im zufriedenstellenden Bereich liegt $(\alpha=0,78)$ (Hossiep 2014). Beide beschriebenen Tests wurden im Papier-Bleistift-Format bearbeitet.

\subsection{Auswertung}

Um die Entwicklung der Einstellungen (Feyerer et al. 2013) und des Pädagogischen Unterrichtswissens (König und Blömeke 2010) in der EG zu untersuchen und mit den Entwicklungen in der KG zu vergleichen, wurden mithilfe der Statistiksoftware SPSS Kovarianzanalysen (ANCOVA) durchgeführt. Dabei wurden die Ergebnisse des ersten Messzeitpunktes $\left(t_{1}\right)$ als Kovariate in die Analyse einbezogen, um den Einfluss des ersten Messzeitpunktes auf die Ergebnisse zum zweiten Messzeitpunkt $\left(t_{2}\right)$ zu berücksichtigen. Die Daten des Follow-ups $\left(t_{3}\right)$ liegen nur für die EG vor. Aufgrund der nunmehr kleinen Fallzahl $(n=14)$ wurden Friedman-Tests als nicht-parametrisches Äquivalent zu einer ANOVA mit Messwiederholung unter Berücksichtigung aller drei Messzeitpunkte durchgeführt (Field 2018). Zusätzlich wurden paarweise Vergleiche zwischen den einzelnen Messzeitpunkten mit einer adjustierten Irrtumswahrscheinlichkeit durchgeführt, um zu überprüfen, ob statistisch überzufällige Lernzuwächse erzielt werden.

\section{Ergebnisse}

Eine ANCOVA zeigt, dass sich das Pädagogische Unterrichtswissen (Gesamtscore) zwischen EG und KG zum zweiten Messzeitpunkt $\left(t_{2}\right)$ unter Kontrolle der Ergebnisse des ersten Messzeitpunktes $\left(t_{1}\right)$ bei einer Irrtumswahrscheinlichkeit von 5\% signifikant unterscheidet $\left(F(1,55)=8,806, p=0,004, \eta^{2}=0,14\right)$. Mit einer Effektgröße von 
$\eta^{2}=0,14$ ist der Effekt als starker Effekt zu interpretieren (Lenhard und Lenhard 2016). In beiden Lehrveranstaltungen ist ein signifikanter Wissenszuwachs zu verzeichnen. Dieses Ergebnis überrascht nicht, da in beiden Lerngruppen aufgrund des Seminarbesuchs im Umfang von vier Semesterwochenstunden ein Wissenszuwachs erwartbar ist. Die ANCOVA zeigt jedoch, dass die EG signifikant mehr Wissen erworben hat (vgl. Abbildung 2). Neben signifikanten Veränderungen des Gesamtscores lassen sich auch statistisch überzufällige Unterschiede zwischen EG und KG für die Dimension Leistungsbeurteilung aufzeigen $\left(F(1,55)=7,515, p=0,008, \eta^{2}=0,12\right)$, die im Seminar insbesondere über eine Auseinandersetzung mit individuellen und kriterialen bzw. sozialen Bezugsnormorientierungen thematisiert wurde.

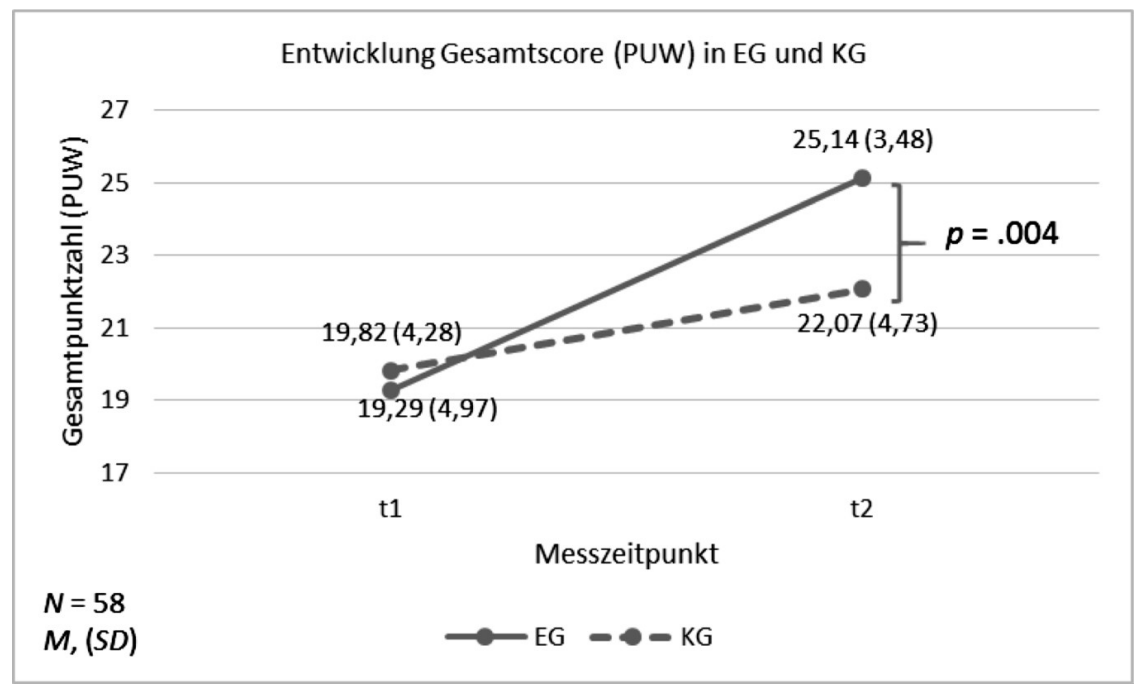

Abbildung 2: Gesamtscore (PUW) in EG und KG ( $\mathrm{t}_{1}$ und $\mathrm{t}_{2}$ )

Für die EG konnte eine signifikante Veränderung des Pädagogischen Unterrichtswissens über die drei Messzeitpunkte hinweg verzeichnet werden $\left(\chi^{2}(2)=10,292, p=0,006\right)$. Dabei sind nur die Veränderungen zwischen dem ersten $\left(M_{t 1}=19,38 ; S D_{t 1}=5,16\right)$ und zweiten Messzeitpunkt $\left(M_{t 2}=25,00 ; S D_{t 2}=3,58\right)$ statistisch bedeutsam $\left(p_{t 1 / t 2}=0,007\right)$, nicht aber zwischen dem zweiten und dritten Messzeitpunkt $\left(M_{t 3}=24,15 ; S D_{t 3}=3,81\right)$ $\left(p_{t 2 / t 3}=0,842\right)$. Das Ergebnis legt nahe, dass die Studierenden das Wissen mindestens mittelfristig erworben haben und nur geringe Vergessenseffekte eintreten.

Neben dem Wissen verändern sich auch die inklusionsbezogenen Einstellungen der Teilnehmer*innen. So unterscheiden sich die Attitudes in der EG zum zweiten Messzeitpunkt unter Kontrolle des ersten Messzeitpunkts signifikant von der KG 
$\left(F(1,50)=10,38, p=0,002, \eta^{2}=0,17\right)$. Die Effektgröße ist mit $\eta^{2}=0,17$ als großer Effekt zu bewerten. Die Haltungen in der EG werden durch die Lehrveranstaltung offenbar positiv beeinflusst, während sich für die KG keine Veränderungen gegenüber dem ersten Messzeitpunkt $\left(t_{1}\right)$ zeigen (vgl. Abbildung 3). Die Attitudes der EG unterscheiden sich zu den drei vorhandenen Messzeitpunkten signifikant $\left(\chi^{2}(2)=10,947\right.$, $p=0,004)$. Die inklusionsbezogenen Einstellungen verändern sich nur zwischen dem ersten $\left(M_{t 1}=2,77 ; S D_{t 1}=0,43\right)$ und zweiten Messzeitpunkt $\left(M_{t 2}=3,25, S D_{t 2}=0,49\right)$ signifikant $\left(p_{t 1 / t 2}=0,009\right)$, nicht aber zwischen dem zweiten und dritten Messzeitpunkt $\left(M_{t 3}=3,16 ; S D_{t 3}=0,41\right)\left(p_{t 2 / 33}=1,000\right)$ bzw. zwischen dem ersten und dritten Messzeitpunkt $\left(p_{t 1 / t 3}=0,099\right)$. Dies legt nahe, dass die Veränderungen der Haltungen gegenüber inklusivem Unterricht in der EG nicht nur kurzfristig eintreten, sondern mindestens mittelfristig stabil bleiben.

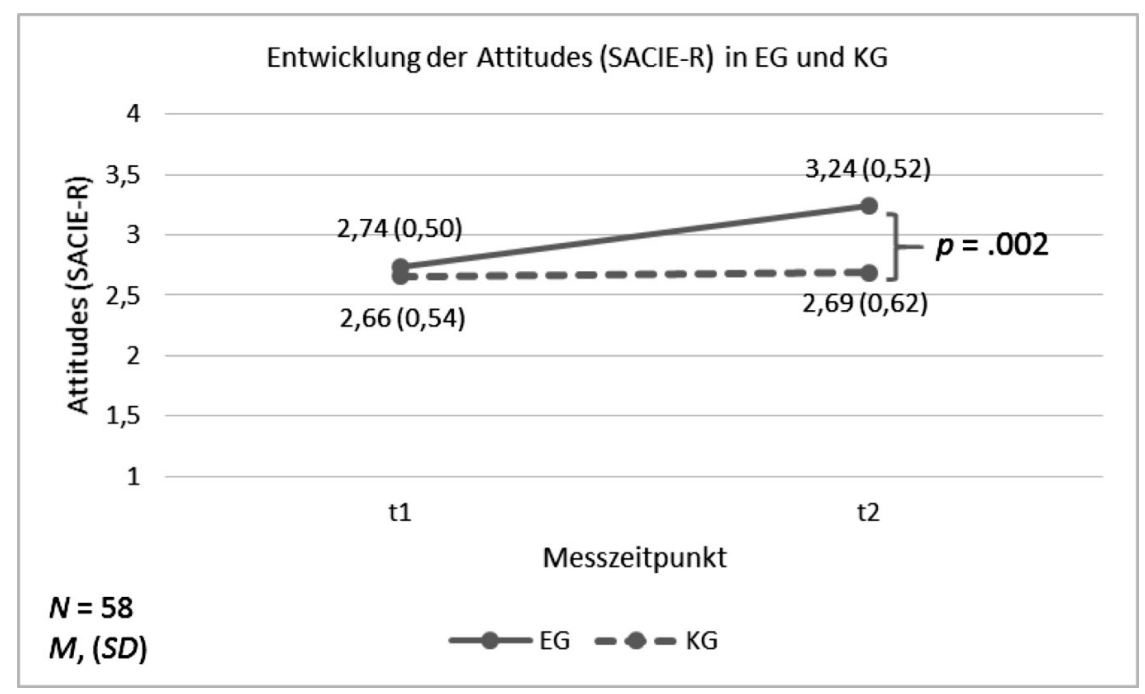

Abbildung 3: Inklusionsbezogene Einstellungen (Attitudes) in EG und KG ( $t_{1}$ und $\left.t_{2}\right)$

Für die Concerns ist für beide Gruppen keine signifikante Veränderung zwischen dem ersten und dem zweiten Messzeitpunkt auszumachen. Die EG weist zu Beginn signifikant geringere Bedenken auf als die $\mathrm{KG}\left(F(1,55)=4,978, p=0,030, \eta^{2}=0,08\right)$. In der EG sinken die Bedenken zwischen dem zweiten $\left(M_{t 2}=2,71 ; S D_{t 2}=0,78\right)$ und dritten Messzeitpunkt $\left(M_{t 3}=2,38, S D_{t 3}=0,58\right)$ noch einmal signifikant ab $\left(\chi^{2}(2)=7,366\right.$, $\left.p=0,025 ; p_{t 2 / t 3}=0,043\right)$. Dieser Befund deutet darauf hin, dass die Studierenden aufgeschlossene, aber nicht unkritische inklusionsbezogene Einstellungen entwickeln (vgl. Abbildung 4). 


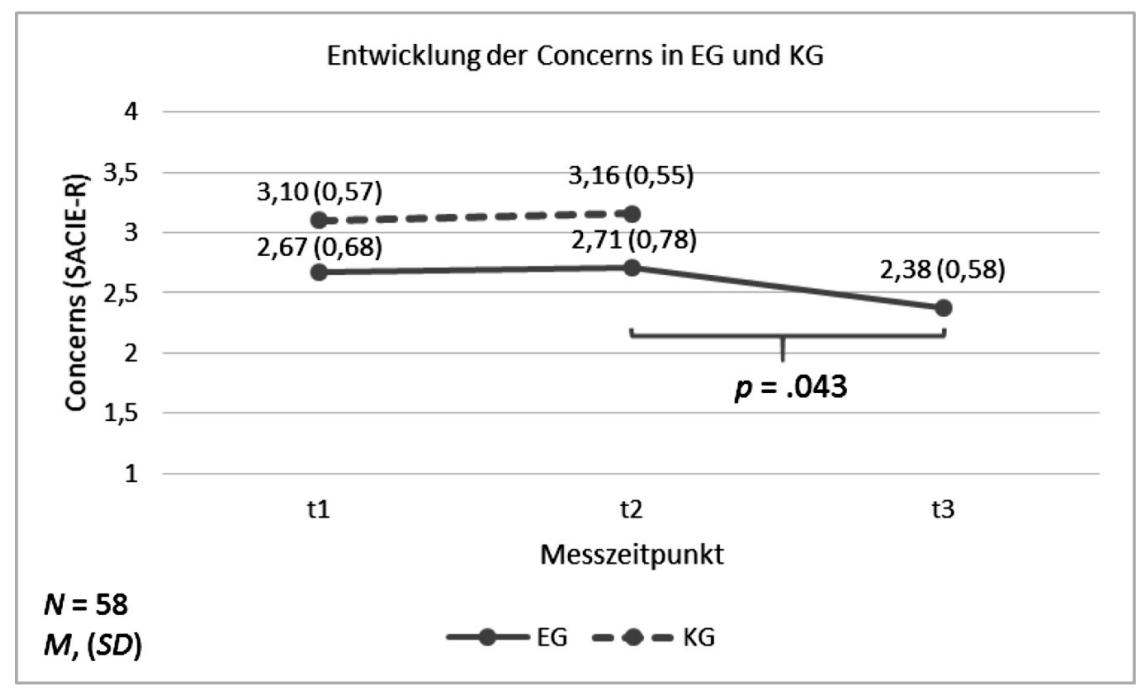

Abbildung 4: Inklusionsbezogene Bedenken (Concerns) in EG $\left(t_{1}-t_{3}\right)$

\section{Diskussion}

In dieser Studie wurde untersucht, ob durch das Seminar Veränderungen bezüglich des bildungswissenschaftlichen Wissens sowie der inklusionsbezogenen Einstellungen erzielt werden können. Es konnte gezeigt werden, dass die Studierenden im Verlauf der Lehrveranstaltung signifikant mehr Wissen erwerben und dieses Wissen sich als mittelfristig stabil erweist.

Komplementär zum Wissen zeigen die Strukturlegepläne, dass die Teilnehmer*innen zu Beginn des Seminars noch einfache Strukturen wählen, am Ende jedoch weitaus komplexere und stärker verdichtete Vernetzungen entstehen. Wie theoretisch erwartet, äußern sich die Studierenden zu Beginn des Seminars oftmals kritisch zu gutem inklusivem Unterricht, wobei sie ihre Positionen überwiegend undifferenziert begründen (»nicht umsetzbar «, »zu aufwendig « etc.). Durch die Vermittlung von Prinzipien guten (inklusiven) Unterrichts im interdisziplinären Teamteaching und deren Verknüpfung mit Einsichten in gelingende inklusive Unterrichtspraxis können die Vorbehalte offenbar deutlich reduziert werden, sodass die Teilnehmenden zu konstruktiveren und aufgeschlosseneren Ansichten gelangen. Insbesondere während der Arbeit am Urteilsraster zeigten sich zu Beginn noch unbestimmte Vorstellungen von Unterrichtsqualität, die sich im weiteren Verlauf jedoch deutlich ausdifferenzierten. Die Beobachtungen legen insgesamt nahe, dass es den Studierenden gelungen ist, ihre Subjektiven Theorien zu ela- 
borieren und mit bildungswissenschaftlichem Wissen anzureichern. Neben elaborierteren (subjektiven) Wissensstrukturen entwickeln sie auch aufgeschlossenere Einstellungen gegenüber inklusivem Unterricht: Die Analyse der Attitudes (SACIE-R) zeigt, dass die Studierenden der EG nach dem Besuch des Seminars und auch zwei Monate danach mittelfristig signifikant positiver gegenüber inklusivem Unterricht eingestellt sind. Während die Concerns unmittelbar nach dem Besuch des Seminars zunächst nahezu unverändert bleiben, ist im Follow-up ein signifikanter Rückgang zu verzeichnen. Die Teilnehmer*innen absolvieren direkt im Anschluss an das Seminar in der Regel ihre Berufsfeldbezogene Praxisstudie, die in Abhängigkeit von ihren Unterrichtsfächern teilweise in der Schule stattfindet. Möglicherweise sammeln sie dabei positive Erfahrungen mit inklusivem Unterricht, die wiederum einen Rückgang ihrer inklusionsbezogenen Bedenken bewirken. Der Zusammenhang von Erfahrungen mit Gemeinsamem Lernen und positiveren inklusionsbezogenen Einstellungen konnte bereits vielfach empirisch belegt werden (z. B. Bosse und Spörer, 2014; Gebhardt et al. 2011; Hellmich und Görel, 2014; Hellmich, Görel und Schwab 2016; Ruberg und Porsch 2017). Darüber hinaus erfahren die Studierenden sich in ihrem Praktikum möglicherweise als selbstwirksam. Studien belegen einen positiven Zusammenhang zwischen Selbstwirksamkeitserwartungen und inklusionsbezogenen Einstellungen (z. B. . Bosse und Spörer, 2014; Bosse et al. 2016; Hecht et al., 2016; Hellmich und Görel 2014; Hellmich, Görel und Schwab, 2016; Knigge und Rotter 2015; Ruberg und Porsch 2017; Urton et al. 2015), sodass die gesteigerten Selbstwirksamkeitserwartungen möglicherweise einen Rückgang der Bedenken bewirken.

Die bisherige Analyse und Interpretation der Daten zeigt, dass sich die Attitudes und Concerns in der EG jeweils unterschiedlich entwickeln: Zum zweiten Messzeitpunkt ist ein signifikanter Anstieg der Attitudes zu verzeichnen, während die Concerns nahezu unverändert bleiben. Offenbar befürworten die Studierenden die gemeinsame Unterrichtung von Kindern mit und ohne sonderpädagogischen Förderbedarf nach dem Seminar stärker als zuvor, sehen inklusiven Unterricht gleichzeitig aber als eine Herausforderung an. Dieser Befund entspricht den Zielen der Lehrveranstaltung: Diese sollte die inklusionsbezogenen Haltungen signifikant positiv beeinflussen, jedoch nicht dazu führen, dass die Studierenden inklusivem Unterricht unkritisch gegenüberstehen bzw. die Anforderungen inklusiven Unterrichts unterschätzen. Die Daten zeigen, dass die Teilnehmenden in Übereinstimmung mit diesen Zielen zu einer realistischen und differenzierten Bewertung von inklusivem Unterricht gelangen. Allerdings ist zu berücksichtigen, dass der SACIE-R Einstellungen zu einem engen Inklusionsverständnis abbildet (Ewing, Monsen und Kielblock 2018), während im Seminar ein weites Inklusionsverständnis angebahnt werden soll (vgl. Abschnitt 2). Perspektivisch wäre daher zu prüfen, wie sich die Einstellungen zu einem weiten Inklusionsverständnis entwickeln.

Die Evaluationsergebnisse deuten darauf hin, dass die im Seminar hergestellte horizontale Kohärenz (innerhalb der Bildungswissenschaften und mit Verknüpfungen zu 
schulpraktischen Erfahrungen in Form von Unterrichtshospitationen) einen Beitrag zur Professionalisierung von angehenden Lehrkräften leisten kann. Einzelne didaktische Elemente - wie die Arbeit am Urteilsraster und die Strukturlegetechnik - haben sich insofern bewährt, als die Studierenden dadurch mit ihren Subjektiven Theorien und Vorannahmen aktiv am Seminar beteiligt werden, sodass sie am Ende zu elaborierteren und differenzierteren Einsichten gelangen. Es bleibt allerdings zu prüfen, ob und inwiefern erworbenes Wissen und inklusionsfreundlichere Einstellungen auch zur Entwicklung von Kompetenzen im Sinne der professionellen Unterrichtswahrnehmung (vgl. Abschnitt 2) beitragen.

Perspektivisch soll das Lehrkonzept in der zweiten Phase der Qualitätsoffensive Lehrerbildung sowohl innerhalb der eigenen Hochschule als auch an andere Hochschulstandorte in Form eines Train-the-Trainer-Konzepts transferiert werden. Hier ist auch die Kooperation mit Vertreter*innen verschiedener Fachdidaktiken geplant, um auf diese Weise die horizontale Kohärenz innerhalb des Lehramtsstudiums weiter auszubauen. Damit soll der Notwendigkeit und Verpflichtung der lehrerbildenden Hochschulen, inklusionssensible Inhalte mit fachdidaktischen Herausforderungen zu verknüpfen, Rechnung getragen werden (Amrhein und Dziak-Mahler 2014; Musenberg und Riegert 2015; KMK und HRK 2015).

\section{Anmerkungen}

1 Für eine detailliertere Beschreibung, wie das Urteilsraster im Seminar realisiert wurde, vgl. Faix et al. 2019b.

2 Biprofessional wird im Rahmen der gemeinsamen Qualitätsoffensive von Bund und Ländern aus Mitteln des Bundesministeriums für Bildung und Forschung gefördert (Förderkennzeichen 01JA1608).

3 An der Universität Bielefeld wird die Zuständigkeit auf Erziehungswissenschaft und Psychologie verteilt, während die Soziologie curricular nicht systematisch an den Bildungswissenschaften beteiligt ist.

4 Ergänzend zum PUW wurde ein weiteres Testinstrument zur standarisierten Erfassung professionellen Wissens zu Inklusion entwickelt. Eine entsprechende Veröffentlichung überschnitt sich mit dem Beginn der Datenerhebung (10/2017). Perspektivisch könnte das Instrument in der zweiten Förderphase unseres Projektes eingesetzt werden (vgl. König et al. 2017; König et al. 2019).

\section{Literatur}

Ajzen, Icek. 2012. »The Theory of Planned Behavior.« In Handbook of Theories of Social Psychology, herausgegeben von Paul A. M. Van Lange, Arie W. Kruglanski und E. Troy Higgins, 439-459. Thousand Oaks: Sage.

Amrhein, Bettina, und Myrle Dziak-Mahler, Hrsg. 2014. Fachdidaktik inklusiv. Auf der Suche nach didaktischen Leitlinien für den Umgang mit Vielfalt in der Schule. Münster: Waxmann.

Avramidis, Elias und Efrosini Kalyva. 2007. »The Influence of Teaching Experience and Professional 
Development on Greek Teachers' Attitudes towards Inclusion." European Journal of Special Needs Education 22, 4: 367-389.

Barth, Victoria L. 2016. Professionelle Wahrnehmung von Störungen im Unterricht. Wiesbaden: Springer.

Bosse, Stefanie, Thorsten Henke, Christian Jäntsch, Jennifer Lambrecht, Miriam Vock,und Nadine Spörer. 2016. »Die Entwicklung der Einstellung zum inklusiven Lernen und der Selbstwirksamkeit von Grundschullehrkräften."Empirische Sonderpädagogik 8, 1: 103-116.

Bosse, Stefanie, und Nadine Spörer. 2014. »Erfassung der Einstellung und der Selbstwirksamkeit von Lehramtsstudierenden zum inklusiven Unterricht.« Empirische Sonderpädagogik 6, 4: 279-299.

Daum, Wolfgang, und Ralf Schneider. 2006. »Interdisziplinäre Lehrveranstaltungen, Studienprojekte und forschendes Lernen. « Journal Hochschuldidaktik 172: 18-20.

Deutsches Institut für Menschenrechte. 2017. "Das Recht auf inklusive Bildung. Allgemeine Bemerkung Nr. 4 des UN-Ausschusses für die Rechte von Menschen mit Behinderungen." Zugriff 29. Mai 2019. https://www.institut-fuer-menschenrechte.de/fileadmin/user_upload/ Publikationen/Information/Information_12_Das_Recht_auf_inklusive_Bildung.pdf

Eagly, Alice H., und Shelly Chaiken. 1993. The Psychology of Attitudes. Fort Worth: Harcourt, Brace \& Janovich.

Ewing, Donna L., Jeremy L. Monsen und Stephan Kielblock. 2018. »Teachers' attitudes towards inclusive education: a critical review of published questionnaires." Educational Psychology in Practice 34, 2: 150-165. https://doi.org/10.1080/02667363.2017.1417822

Faix, Ann-Christin, Birgit Lütje-Klose, Annette Textor und Elke Wild. 2019a. »Strukturlegepläne als hochschuldidaktisches Instrument zur Evaluation und Reflexion Subjektiver Theorien."Herausforderung Lehrer_innenbildung [angenommen].

Faix, Ann-Christin, Birgit Lütje-Klose, Annette Textor und Elke Wild. 2019b. »Ist das guter inklusiver Unterricht? Mit Videoanalysen und Hospitationen von der Theorie zur Praxisreflexion.« Herausforderung Lehrer_innenbildung 2, 3: 1-19. https://doi.org/10.4119/hlz-2462

Feyerer, Ewald, Harald Reibnegger, Petra Hecht, Katharina Soukup-Altrichter, Christine Plaimauer, Eva Prammer-Semmler, Irene Moser und Sabine Bruch. 2013. Inklusive Bildung. Einstellungen und Kompetenzen von Lehramtsstudierenden und LehrerInnen für die Umsetzung inklusiver Bildung. Deutsche Version SACIE-R 2013 \& TEIP 2013. https://www.psycharchives.org/bitstream/ 20.500.12034/467/1/PT_9007118_SACIE-R_TEIP_Fragebogen.pdf

Field, Andrew. 2018. Discovering Statistics Using IBM SPSS Statistics. Thousand Oaks: Sage.

Fives, Helenrose, und Michelle M. Buehl. 2012. „Spring cleaning for the smessy، contruct of teachers' beliefs: What are they? Which have been examined? What can they tell us? « In APA educational psychology handbook, Vol. 2: Individual differences and cultural and contextual factors, herausgegeben von Karen R. Harris, Steve Graham und Tim Urdan, 471-499. Washington, D. C.: American Psychological Association.

Gebhardt, Markus, Susanne Schwab, Hannelore Reicher, Barbara Ellmeier, Sonja Gmeiner, Peter Rossmann und Barbara Gasteiger-Klicpera. 2011. „Einstellungen von LehrerInnen zur schulischen Integration von Kindern mit einem sonderpädagogischen Förderbedarf in Österreich." Empirische Sonderpädagogik 3, 4: 275-290.

Goeze, Annika. 2010. »Was ist ein guter Fall? Kriterien für die Entwicklung und Auswahl von Fällen für den Einsatz in der Aus- und Weiterbildung. «In Mediengestützte Fallarbeit:Konzepte, Erfahrungen und Befunde zur Kompetenzentwicklung von Erwachsenenbildnern, herausgegeben von Josef Schrader, Reinhard Hohmann und Stefanie Hartz, 125-145. Bielefeld: Bertelsmann.

Gold, Bernadette, Stephan Förster und Manfred Holodynski. 2013. „Evaluation eines videobasier- 
ten Trainingsseminars zur Förderung der professionellen Wahrnehmung von Klassenführung im Grundschulunterricht."Zeitschrift für Pädagogische Psychologie 27, 3: 141-155.

Groeben, Norbert, Diethelm Wahl, Jörg Schlee und Brigitte Scheele. 1988. Das Forschungsprogramm Subjektive Theorien: eine Einführung in die Psychologie des reflexiven Subjekts. Tübingen: Francke.

Hecht, Petra, Claudia Niedermair und Ewald Feyerer. 2016. »Einstellungen und inklusionsbezogene Selbstwirksamkeitsüberzeugungen von Lehramtsstudierenden und Lehrpersonen im Berufseinstieg. Messverfahren und Befunde aus einem Mixed-Methods-Design."Empirische Sonderpädagogik 8, 1: 86-102.

Hellermann, Christina, Bernadette Gold und Manfred Holodynski. 2015. »Förderung von Klassenführungsfähigkeiten im Lehramtsstudium. Die Wirkung der Analyse eigener und fremder Unterrichtsvideos auf das strategische Wissen und die professionelle Wahrnehmung."Zeitschrift für Entwicklungspsychologie und Pädagogische Psychologie 47, 2: 97-109.

Hellmann, Katharina. 2019. »Kohärenz in der Lehrerbildung - Theoretische Konzeptualisierung.« In Kohärenz in der Lehrerbildung. Theorien, Modelle und empirische Befunde, herausgegeben von Katharina Hellmann, Jessica Kreutz, Martin Schwichow und Katja Zaki, 9-30. Wiesbaden: Springer.

Hellmich, Frank, und Gamze Görel. 2014. »Erklärungsfaktoren für Einstellungen von Lehrerinnen und Lehrern zum inklusiven Unterricht in der Grundschule.« Zeitschrift für Bildungsforschung 4, 3: 227-240.

Hellmich, Frank, Gamze Görel und Susanne Schwab. 2016. „Einstellungen und Motivation von Lehramtsstudentinnen und -studenten in Bezug auf den inklusiven Unterricht in der Grundschule - Ein Vergleich zwischen Deutschland und Österreich.« Empirische Sonderpädagogik 8, 1:67-85.

Hossiep, Rüdiger. 2014. „Cronbachs Alpha.» In Dorsch - Lexikon der Psychologie, herausgegeben von Markus A. Wirtz, 343. Bern: Hogrefe.

KMK. 2004. Standards für die Lehrerbildung: Bildungswissenschaften. Zugriff 29. Mai 2019. https:// www.kmk.org/fileadmin/veroeffentlichungen_beschluesse/2004/2004_12_16-Standards -Lehrerbildung.pdf.

KMK und HRK. 2015. Lehrerbildung für eine Schule der Vielfalt. Gemeinsame Empfehlung von Hochschulrektorenkonferenz und Kultusministerkonferenz. Beschluss der Kultusministerkonferenz vom 12.03.2015/Beschluss der Hochschulrektorenkonferenz vom 18.03.2015. Zugriff 29. Mai 2019. https://www.kmk.org/fileadmin/Dateien/veroeffentlichungen_beschluesse/2015/ 2015_03_12-Schule-der-Vielfalt.pdf.

König, Johannes, und Sigrid Blömeke. 2009. »Pädagogisches Wissen von angehenden Lehrkräften. Erfassung und Struktur von Ergebnissen der fachübergreifenden Lehrerausbildung." Zeitschrift für Erziehungswissenschaft 12: 499-527.

König, Johannes, und Sigrid Blömeke. 2010. Pädagogisches Unterrichtswissen (PUW). Dokumentation der Kurzfassung des TEDS-M Testinstruments zur Kompetenzmessung in der ersten Phasen der Lehrerbildung. Berlin: Humboldt-Universität.

König, Johannes, Kristina Gerhard, Kai Kaspar und Conny Melzer. 2019. »Professionelles Wissen von Lehrkräften zur Inklusion: Überlegungen zur Modellierung und Erfassung mithilfe standardisierter Testinstrumente."Pädagogische Rundschau 73, 1: 43-64.

König, Johannes, Kristina Gerhard, Conny Melzer, Anna-Maria Rühl, Julie Zenner und Kai Kaspar. 2017. »Erfassung von pädagogischem Wissen für inklusiven Unterricht bei angehenden Lehrkräften: Testkonstruktion und Validierung. « Unterrichtswissenschaft 45, 4: 223-242.

Knigge, Michel, und Carolin Rotter. 2015. „Unterrichtsplanungen bei Lehramtsstudierenden im Falle der Wahrnehmung von vermeintlich sbesonderen Schülerinnen und Schülern und 
ihr Zusammenhang mit Selbstwirksamkeitsüberzeugungen und Einstellungen in Bezug zu Inklusion - beispielhafte Mixed-Methods-Analysen aus der EiLInk-Studie."Empirische Sonderpädagogik 7, 3: 223-240.

Kullmann, Harry, Birgit Lütje-Klose, Annette Textor, Jutta Berard und Katharina Schitow. 2015. »lnklusiver Unterricht - (Auch) eine Frage der Einstellung!: Eine Interviewstudie über Einstellungen und Bereitschaften von Lehrkräften und Schulleitungen zur Inklusion. «In Schriftenreihe Theorie und Praxis der Schulpädagogik. Vol. 28. Inklusive Unterrichtspraxis und -entwicklung, herausgegeben von Catrin Siedenbiedel und Caroline Theurer, 181-196. Immenhausen bei Kassel: Prolog.

Kunina-Habenicht, Olga, Hendrik Lohse-Bossenz, Mareike Kunter, Theresa Dicke, Doris Förster, Jill Gößling und Franziska Schulze-Stocker. 2012. „Welche bildungswissenschaftlichen Inhalte sind wichtig in der Lehrerbildung? Ergebnisse einer Delphi-Studie."Zeitschrift für Erziehungswissenschaft 15, 4: 649-682.

Kunter, Mareike, Jürgen Baumert, Werner Blum, Uta Klusmann, Stefan Krauss und Michael Neubrand, Hrsg. 2011. Professionelle Kompetenz von Lehrkräften. Ergebnisse des Forschungsprogramms COACTIV. Münster: Waxmann.

Lattuca, Lisa R., Lois J. Voight und Kimberly Q. Fath. 2004. „Does Interdisciplinarity Promote Learning? Theoretical Support und Reserachable Questions." The Review of Higher Education 28, 1: 23-48.

Lenhard, Wolfang, und Alexandra Lenhard. 2016. Berechnung von Effektstärken. https://www. psychometrica.de/effektstaerke.html. Dettelbach: Psychometrica. DOI: 10.13140/RG.2.1. 3478.4245

Lindmeier, Christian, und Birgit Lütje-Klose. 2018. »Inklusion.« In Handbuch Schulpädagogik, herausgegeben von Marius Harring, Michaela Gläser-Zikuda und Carsten Rohlfs, 43-53. Münster: Waxmann.

Loreman, Tim, Chris Earle, Umesh Sharma und Chris Forlin. 2007. »The Development of an Instrument for Measuring Pre-Service Teachers' Sentiments, Attitudes and Concerns about Inclusive Education. «International Journal of Special Education 22, 2, 150-159.

Lütje-Klose, Birgit, Susanne Miller und Holger Ziegler. 2014. »Professionalisierung für die inklusiver Schule als Herausforderung für die Lehrerlnnenbildung."Soziale Passagen 6, 1:69-84.

Lütje-Klose, Birgit, Phillip Neumann und Bettina Stresse. 2017. »Schulische Inklusion in NordrheinWestfalen (NRW) - sieben Jahre nach Ratifizierung der UN-BRK.« Zeitschrift für Inklusion 11, 2: o. S. http://www.inklusion-online.net/index.php/inklusion-online/article/view/416.

Moser, Vera, Jan Kuhl, Hubertus Redlich und Lea Schäfer. 2014. »Beliefs von Studierenden sonderund grundschulpädagogischer Studiengänge.« Zeitschrift für Erziehungswissenschaft 17, 4: 661-678. https://doi.org/10.1007/s11618-014-0587-1

Moser, Vera. 2018. »Lehrerinnen- und Lehrerbildung.« In Handbuch schulische Inklusion, herausgegeben von Tanja Sturm und Monika Wagner-Willi, 289-298. Opladen und Toronto: Barbara Budrich.

Musenberg, Oliver, und Judith Riegert. 2015. »Inklusiver Fachunterricht als didaktische Herausforderung." In Inklusiver Fachunterricht in der Sekundarstufe, herausgegeben von Judith Riegert und Oliver Musenberg, 13-28. Stuttgart: Kohlhammer.

Petko, Dominik, Doreen Prasse und Kurt Reusser. 2014. „Online-Plattformen für die Arbeit mit Unterrichtsvideos: Eine Übersicht.« Beiträge zur Lehrerinnen- und Lehrerbildung 32, 2: 247-261.

Piezunka, Anne, Cornelia Gresch und Michael Wrase. 2018. „Artikel 24 der UN-Behindertenrechtskonvention."Zeitschrift für Inklusion 12, 1. https://www.inklusion-online.net/index. php/inklusion-online/article/view/369

Reusser, Kurt, und Christine Pauli. 2014. »Berufsbezogene Überzeugungen von Lehrerinnen und 
Lehrern." In Handbuch der Forschung zum Lehrerberuf, herausgegeben von Ewald Terhart, Hedda Bennewitz und Martin Rothland, 642-661. Münster: Waxmann.

Ruberg, Christiane, und Raphaela Porsch. 2017. „Einstellungen von Lehramtsstudierenden und Lehrkräften zur schulischen Inklusion. Ein systematisches Review deutschsprachiger Forschungsarbeiten. « Zeitschrift für Pädagogik 63, 1: 393-416.

Scheele, Brigitte, Norbert, Groeben und Ursula Christmann. 1992. »Ein alltagssprachliches Struktur-Lege-Spiel als Flexibilisierungsversion der Dialog-Konsens-Methodik.« In Struktur-LegeVerfahren als Dialog-Konsens-Methodik. Ein Zwischenfazit zur Forschungsentwicklung bei der rekonstruktiven Erhebung Subjektiver Theorien, herausgegeben von Brigitte Scheele, 152-195. Münster: Aschendorff.

Schulgesetz NRW. 2018. Schulgesetz für das Land Nordrhein-Westfalen. Zugriff 29. Mai 2019. https:// www.schulministerium.nrw.de/docs/Recht/Schulrecht/Schulgesetz/Schulgesetz.pdf

Seidel, Tina, Geraldine Blomberg und Kathleen Stürmer. 2010. »Observer«. Validierung eines videobasierten Instruments zur Erfassung der professionellen Wahrnehmung von Unterricht.« In Kompetenzmodellierung. Zwischenbilanz des DFG-Schwerpunktprogramms und Perspektiven des Forschungsansatzes (Zeitschrift für Pädagogik. Beiheft 56), herausgegeben von Eckhard Klieme, Detlev Leutner und Martina Kenk, 296-306. Weinheim, Beltz.

Seidel, Tina, und Kathleen Stürmer. 2014. »Modeling and Measuring the Structure of Professional Vision in Preservice Teachers. "American Educational Research Journal 51, 4, 739-771. https:// doi.org/10.3102/0002831214531321

Seidel, Tina, und Felicitas Thiel. 2017. "Standards und Trends der videobasierten Lehr-Lernforschung." In Videobasierte Unterrichtsforschung. Analysen von Unterrichtsqualität, Gestaltung von Lerngelegenheiten und Messung professionellen Wissens. Zeitschrift für Erziehungswissenschaft. Sonderheft 32, herausgegeben von Tina Seidel und Felicitas Thiel, 1-21. Wiesbaden: Springer. https://doi.org/10.1007/978-3-658-15739-5_1

Sherin, Miriam G. 2007. »The development of teachers' professional vision in video clubs. «In Video research in the learning sciences, herausgegeben von Ricki Goldman, Roy Pea, Brigid Barron und Sharon J. Derry, 383-395. Mahwah: Erlbaum.

Sherin, Miriam G., und Elizabeth A. van Es. 2009. »Effects of Video Club Participation on Teachers' Professional Vision." Journal of Teacher Education 60, 1: 20-37.

Shulman, Lee S. 1986. »Those Who Understand: Knowledge Growth in Teaching.« Educational Researcher 15, 2: 4-14.

Smith Ducoffe, Sandra J., Cheryl L. Tromley und Michael Tucker. 2006. »Interdisciplinary, TeamTaught, Undergraduate Business Courses: The Impact of Integration. « Journal of Management Education 30, 2: 276-294.

Terhart, Ewald, Franziska Schulze-Stocker, Olga Kunina-Habenicht, Theresa Dicke, Doris Förster, Hendrik Lohse-Bossenz, Jill Gößling, Mareike Kunter, Jürgen Baumert und Detlev Leutner. 2012. »Bildungswissenschaftliches Wissen und der Erwerb professioneller Kompetenz in der Lehramtsausbildung. Eine Kurzdarstellung des BilWiss-Projekts." Lehrerbildung auf dem Prüfstand 5, 1: 96-106.

Textor, Annette, Harry Kullmann und Birgit Lütje-Klose. 2014. »Eine Inklusion unterstützende Didaktik. Rekonstruktionen aus der Perspektive inklusionserfahrener Lehrkräfte.» In Jahrbuch für Allgemeine Didaktik 2014, herausgegeben von Klaus Zierer, 69-91. Baltmannsweiler: Hohengehren.

UN-BRK. 2008. Die UN-Behindertenrechtskonvention. Übereinkommen über die Rechte von Menschen mit Behinderung. Bundesgesetzblatt Teil II Nr. 35. Zugriff 29. Mai 2019. http://www.un.org/ Depts/german/uebereinkommen/ar61106-dbgbl.pdf. 1419-1457.

Urton, Karolina, Jürgen Wilbert und Thomas Hennemann. 2015. »Die Einstellung zur Integration 
und die Selbstwirksamkeit von Lehrkräften."Psychologie in Erziehung und Unterricht 62, 2: 147-157.

Wahl, Diethelm. 2001. »Wissen sichtbar machen (1). Nachhaltig lernen mit der Struktur-Lege-Technik.«Praxis Schule 5-10 5, 63-65.

Wahl, Diethelm. 2013. Lernumgebungen erfolgreich gestalten. Vom trägen Wissen zum kompetenten Handeln. Bad Heilbrunn: Klinkhardt.

Werning, Rolf, und Birgit Lütje-Klose. 2016. Einführung in die Pädagogik bei Lernbeeinträchtigungen. München: Reinhardt.

Wilde, Annett, und Mareike Kunter. 2016. „Überzeugungen von Lehrerinnen und Lehrern."In Beruf Lehrer/Lehrerin. Ein Studienbuch, herausgegeben von Martin Rothland, 299-315. Münster: Waxmann.

Wirtz, Markus, und Franz Caspar. 2002. Beurteilerübereinstimmung und Beurteilerreliabilität. Göttingen: Hogrefe.

\section{Die Autorinnen}

Ann-Christin Faix, M. Ed., ist wissenschaftliche Mitarbeiterin an der Universität Bielefeld, Fakultät für Psychologie und Sportwissenschaft, AE09 Pädagogische Psychologie.

Kontakt:a.faix@uni-bielefeld.de

Elke Wild, Prof. Dr., ist Hochschullehrerin an der Universität Bielefeld, Fakultät für Psychologie und Sportwissenschaft, AE09 Pädagogische Psychologie.

Kontakt: elke.wild@uni-bielefeld.de

Birgit Lütje-Klose, Prof. Dr., ist Hochschullehrerin an der Universität Bielefeld, Fakultät für Erziehungswissenschaft, AG3 Schultheorie mit dem Schwerpunkt Grund- und Förderschulen.

Kontakt: birgit-luetje@uni-bielefeld.de

Annette Textor, Prof. Dr., ist Hochschullehrerin an der Universität Bielefeld, Fakultät für Erziehungswissenschaft, AG4 Schulentwicklung und Schulforschung.

Kontakt: annette.textor@uni-bielefeld.de 